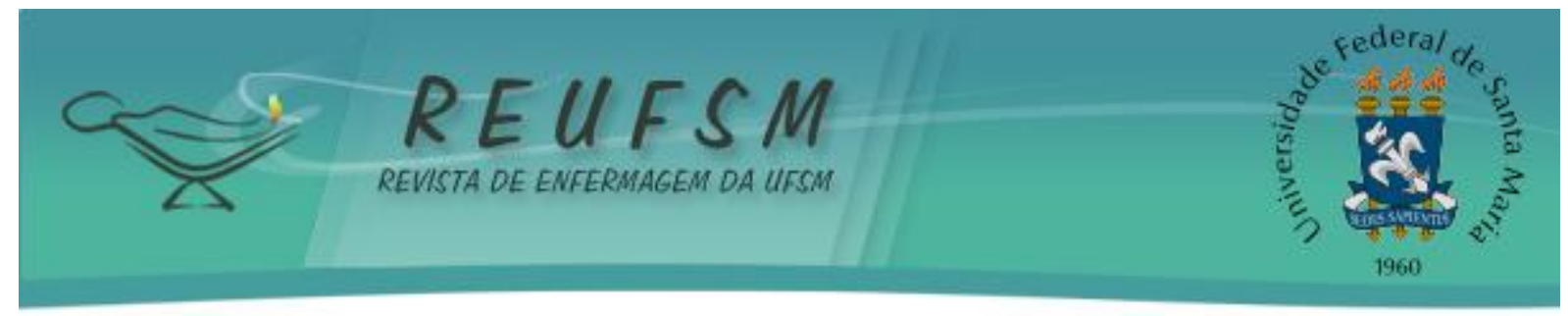

ARTIGO ORIGINAL

\title{
DIAGNÓSTICOS DE ENFERMAGEM EM PACIENTES HIPERTENSOS ACOMPANHADOS EM AMBULATÓRIO MULTIPROFISSIONAL
}

\author{
NURSING DIAGNOSIS IN HYPERTENSIVE PATIENTS FOLLOWED IN MULTIDISCIPLINARY \\ AMBULATORY
}

\section{DIANÓSTICOS DE ENFERMERÍA EN PACIENTES HIPERTENSOS ACOMPAÑADOS EN UN AMBULATORIO MULTIPROFESIONAL}

\author{
Danielle Plocharski Calegari ${ }^{1}$ \\ Silvia Goldmeier ${ }^{2}$ \\ Maria Antonieta Moraes ${ }^{3}$ \\ Emiliane Nogueira de Souza ${ }^{4}$
}

RESUMO: Objetivo: descrever a prevalência de diagnósticos de enfermagem em hipertensos em ambulatório multiprofissional. Métodos: estudo transversal com hipertensos acompanhados por médico, enfermeiro, fisioterapeuta, psicólogo e nutricionista em hospital cardiológico. Durante consulta de enfermagem, foram identificados os diagnósticos de enfermagem de acordo com a taxonomia da NANDA-I. Resultados: avaliados 65 pacientes, $73,8 \%$ mulheres, idade $55,3 \pm 13,1$ anos, tempo de hipertensão arterial sistêmica de $12,6 \pm 9,4$ anos, $27,7 \%$ diabéticos e $32,3 \%$ com apnéia do sono. Diagnósticos de enfermagem prevalentes: Disposição para controle aumentado do regime terapêutico e Ansiedade (50,8\%), Estilo de vida sedentário (47,7\%) e Padrão de sono prejudicado (46,4\%). Estes relacionados aos domínios: promoção da saúde, enfrentamento e tolerância ao stress e atividade e repouso compatíveis com a doença crônica e à complexidade do tratamento. Conclusão: diagnósticos de enfermagem fornecem subsídios para autocuidado e identificam a melhor conduta entre pacientes com regimes terapêuticos mais complexos.

Descritores: Diagnóstico de enfermagem; Hipertensão; Assistência ambulatorial.

ABSTRACT: Objective: to describe the prevalence of nursing diagnoses in hypertensive patients followed in multidisciplinary outpatient clinic. Methods: cross sectional study with hypertensive followed by physicians, nurses, physiotherapists, psychologists, and nutritionists in a cardiology hospital. During nursing consultation nursing diagnoses were identified according to the taxonomy of NANDA I. Results: study assessed 65 patients, $73.8 \%$ were women, $55.3 \pm 13.1$ years, duration of hypertension of $12.6 \pm 9.4$ years, $27.7 \%$ diabetes, and $32.3 \%$ had sleep apnea.Prevalent nursing diagnoses: Disposition for increased control of the therapeutic regimen and Anxiety (50.8\%), sedentary lifestyle (47.7\%), and impaired sleep pattern (46.4\%). These related the domains: health promotion, coping and stress tolerance, activity and rest compatible with chronic illness and complexity of treatment Conclusion: Nursing diagnoses may provide information for self care and to identify the best treatment for patients with more complex treatment regimen.

\footnotetext{
${ }^{1}$ Enfermeira, Especialista em Cardiologia, Residente no Instituto de Cardiologia/Fundação Universitária de Cardiolgia - Porto Alegre, RS. E-mail: dsgbos@gmail.com

${ }^{2}$ Enfermeira, Doutora em Ciências da Saúde: Cardiologia, Professora do Pós-Graduação Lato-Sensu Enfermagem em Cardiologia do Instituto de Cardiologia/Fundação Universitária de Cardiolgia - Porto Alegre, RS. E-mail: silvia.gold@cardiologia.org.br

${ }^{3}$ Enfermeira, Doutora em Ciências da Saúde: Cardiologia, Professora do Pós-Graduação Lato-Sensu Enfermagem em Cardiologia do Instituto de Cardiologia/Fundação Universitária de Cardiolgia - Porto Alegre, RS. E-mail: moraes.enf@cardiologia.org.br

${ }^{4}$ Enfermeira, Doutora em Ciencias da Saúde: Cardiologia e Ciências Cardiovasculares, Professora Adjunta da Universidade Federal de Ciências da Saúde de Porto Alegre - Porto Alegre, RS. E-mail: enogsouza@hotmail.com
} 


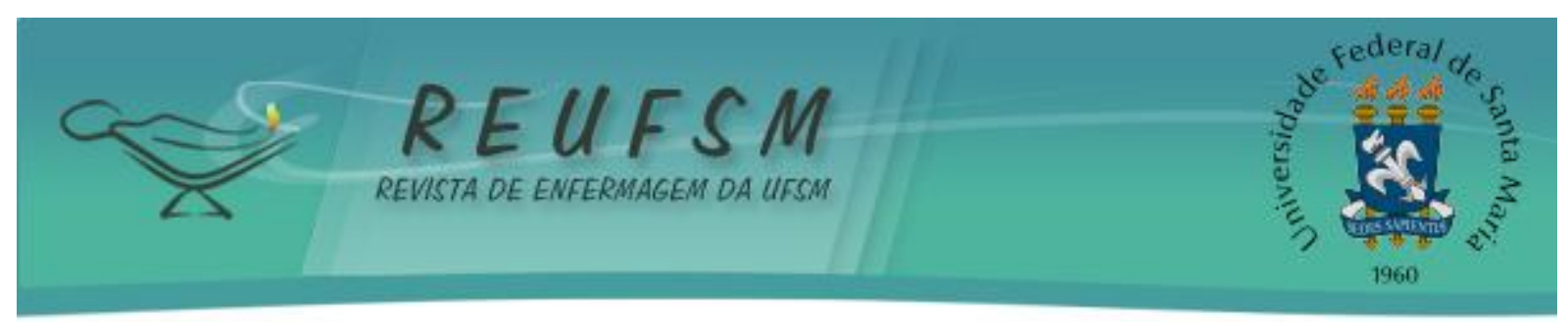

Descriptors: Nursing diagnosis; Hypertension; Outpatient clinic attending.

RESUMEN: Objetivo: describir prevalencia de diagnósticos de enfermería en hipertensos en ambulatoria multidisciplinaria. Metodos: estudio descriptivo transversal con hipertensos acompañada por médico, enfermería, fisioterapia, psicologia y nutricionista en hospital de cardiología. Durante la consulta de la enfermería, diagnósticos de enfermería se identificaron de acuerdo con la taxonomía de NANDA-I. Resultados: Evaluó 65 pacientes, 73,8\% mujeres, edad 55,3 \pm 13,1 años, tiempo de hipertensión 12,6 \pm 9,4 años , 27,7\% diabéticos y 32,3\% con apnea del sueño. Diagnósticos de enfermería prevalencia: Provisión para un control del régimen terapéutico y la ansiedad (50,8\%), sedentarismo $(47,7 \%)$ y alteración de los patrones del sueño $(46,4 \%)$. Estas se refieren a campos a la promoción de la salud, afrontamiento y tolerancia al estrés, actividad y descanso, de conformidad con enfermedades crónicas y complejidad del tratamiento. Conclusión: diagnósticos de enfermería subvenciona el auto-cuidado e identifica mejores prácticas para pacientes con régimen de tratamiento más complejo.

Descriptores: Diagnóstico de Enfermería; Hipertensión; Consulta externa.

\section{INTRODUÇÃO}

A hipertensão arterial sistêmica (HAS) é a mais frequente das doenças cardiovasculares. É também o principal fator de risco para complicações como acidente vascular cerebral e infarto agudo do miocárdio, além da doença renal crônica terminal. Estima-se que entre os 12 milhões de hipertensos do Brasil, apenas 10\% são tratados efetivamente. ${ }^{1} 0$ mesmo quadro pode ser identificado em diferentes faixas etárias, sendo que nos indivíduos idosos o índice de hipertensos chega a $65 \%^{2}$, havendo, igualmente, uma prevalência considerável em crianças e adolescentes. ${ }^{3}$

O manejo da HAS é complexo, mesmo em ambulatórios de referência o controle pressórico é insatisfatório, havendo um número expressivo de pacientes com pressão não controlada. ${ }^{4}$ Alguns estudos demonstraram que cerca da metade dos hipertensos não apresentam a pressão arterial controlada. ${ }^{5-7}$ Entre as causas mais citadas para hipertensão não-controlada está a má adesão ao tratamento. ${ }^{8}$ A adesão ao tratamento corresponde ao grau de seguimento do paciente a determinada prescrição/orientação ${ }^{9}$, sendo compreendida como problema multifatorial. ${ }^{10}$ As taxas de adesão são mais altas em pacientes com condições agudas, quando comparado a portadores de doenças crônicas. A persistência entre os pacientes crônicos é muito baixa, diminuindo drasticamente após os primeiros seis meses de terapia. ${ }^{9}$

Quanto mais elevada pressão arterial, maior a probabilidade de desenvolvimento prematuro de doenças cardiovasculares por aterosclerose acelerada, marca da hipertensão não controlada. Quando não tratados, cerca de $50 \%$ dos pacientes morrem de doença arterial coronariana ou insuficiência cardíaca congestiva e aproximadamente $33 \%$ de acidente vascular encefálico, e 10 a $15 \%$ de insuficiência renal. ${ }^{11}$

A HAS, por ser multifatorial, necessita de abordagem multiprofissional para atingir melhores resultados. ${ }^{12}$ Entre os profissionais atuantes no cuidado aos hipertensos estão os enfermeiros. A responsabilidade do cuidar, realizado pela enfermagem, exige que as suas decisões sobre as intervenções propostas sejam fundamentadas na avaliação do estado de saúde do indivíduo. Para realizar esta avaliação, é importante que se utilize o processo de enfermagem, pois por meio dele é possível identificar as necessidades de cuidado ao paciente de forma individual e integral. ${ }^{13}$

A aplicação do processo de enfermagem (PE) possibilita ao enfermeiro a prestação de cuidados individualizados, centrado nas necessidades humanas básicas. ${ }^{14} \mathrm{O}$ PE promove o diagnóstico das necessidades do cliente, garante a prescrição adequada dos cuidados, a 


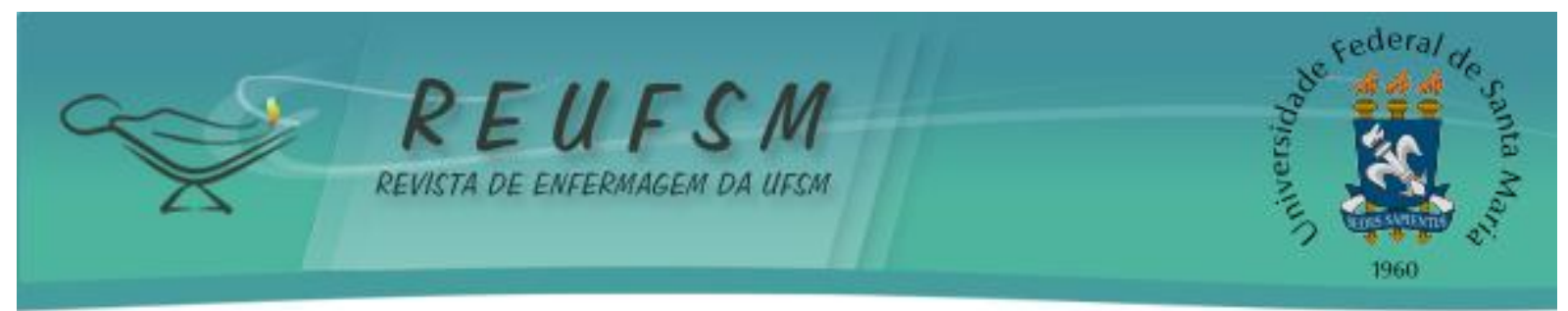

avaliação dos resultados e qualidade da assistência prestada. ${ }^{15}$ Profissionais pesquisadores da área da enfermagem dedicados ao estudo da sistematização da assistência de enfermagem, nos mais diversos campos de atuação, afirmam que há estreita relação entre a aplicação deste método com a humanização e a qualidade do cuidado. ${ }^{16}$ Sendo assim o diagnóstico de enfermagem (DE) é um julgamento clínico das respostas do indivíduo, família e comunidade, aos problemas de saúde reais ou potenciais, proporcionando a base para a seleção das intervenções de enfermagem, visando o alcance dos resultados pelos quais a enfermeira é responsável. ${ }^{17}$

Para realizar as atividades do cuidado, o enfermeiro necessita de instrumental conceitual e técnico para abordar a realidade prática. O método empregado é a organização, a sistemática racional de ações para alcançar os objetivos da assistência cuja ferramenta é a Sistematização da Assistência de Enfermagem (SAE). ${ }^{18}$

Tendo em vista que a HAS é uma doença de difícil manejo e seu descontrole representa alto risco cardiovascular para seus portadores, a abordagem contínua da enfermagem aos pacientes crônicos apresenta efeitos relevantes na prevenção primária e secundária de eventos cardiovasculares.

A identificação dos diagnósticos de enfermagem (DE) fornece subsídios ao enfermeiro, para a elaboração de um plano de cuidados mais específico, de acordo com o comprometimento de cada indivíduo. A partir do conhecimento de tais respostas humanas e de seus respectivos fatores preditores, torna-se possível predizer, prever, detectar e controlar as complicações potenciais. ${ }^{19}$

Para qualificar a intervenção do enfermeiro nos diferentes cenários de cuidados torna-se necessária a identificação do DE. Assim, este estudo tem por objetivo identificar os diagnósticos de enfermagem prevalentes em ambulatório de HAS.

\section{MÉTODOS}

Estudo transversal com abordagem quantitativa, realizado com pacientes hipertensos em acompanhamento ambulatorial em hospital de referência cardiológica do Rio Grande do Sul, no período de abril de 2010 a abril de 2011. Foram incluídos pacientes com HAS, com mais de 18 anos de idade, de ambos os sexos, que aceitaram participar do estudo.

\section{Logística do Estudo}

No ambulatório de hipertensão o atendimento é realizado duas vezes por semana, totalizando quatro pacientes por dia. Os pacientes que recebem encaminhamento são atendidos pela equipe multiprofissional (médico cardiologista, enfermeira, nutricionista, fisioterapeuta e psicóloga).

A consulta de enfermagem foi realizada por uma enfermeira, pós-graduada em nível strito sensu em cardiologia, acompanhada por uma residente de enfermagem em cardiologia, que consistia na anamnese, no exame físico, na identificação dos diagnósticos de enfermagem e orientações. As avaliações dos demais profissionais da equipe foram realizadas de maneira individual, logo após a avaliação da enfermagem. A coleta de dados foi realizada durante a consulta de enfermagem utilizando-se formulário específico, no qual constavam os DE (taxonomia NANDA-I) e características definidoras. Os DE mais prevalentes foram atribuídos pela enfermeira quando 0 paciente apresentava pelo menos duas características definidoras. Foi considerado como DE de alta prevalência quando os índices do DE atingissem 45-60\%, o de média prevalência quando os índices atingissem de $30-44 \%$ e os de baixa prevalência quando os índices fossem abaixo de $30 \%$. 


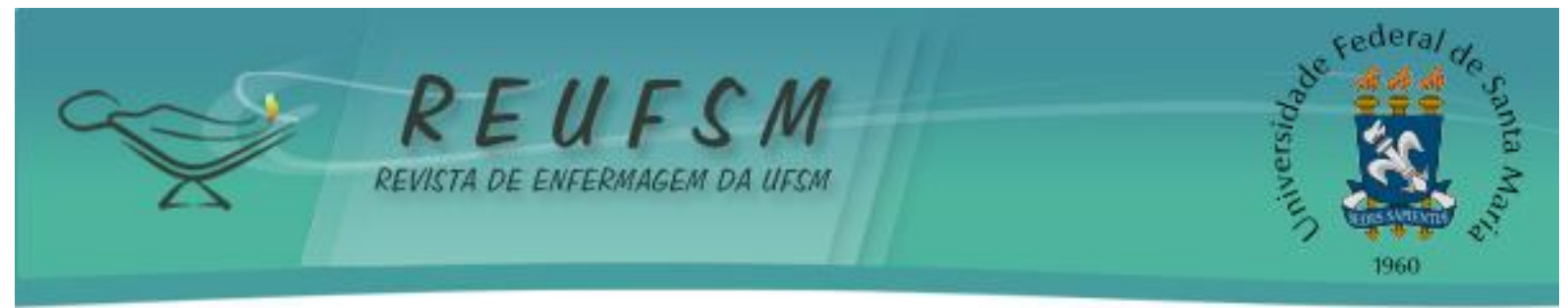

\section{Considerações Éticas}

Esta pesquisa foi conduzida de acordo com os padrões éticos exigidos, segundo a Resolução 196/96. O estudo foi aprovado pelo Comitê de Ética em Pesquisa, sob número 4449/10 em 28 de abril de 2010. Todos os participantes assinaram o Termo de Consentimento Livre e Esclarecido.

\section{Análise Estatística}

A análise dos dados foi realizada através de software SPSS ${ }^{\circledR}$ for Windows versão 19.0. Foi utilizado o teste de Qui-quadrado para avaliar associação entre variáveis categóricas e teste $t$ de Student para variáveis contínuas. Foi utilizado uma população de acesso totalizando 65 pacientes.

\section{RESULTADOS}

Foram incluídos 65 pacientes, com idade média de 54,3 $\pm 13,1$ anos, e 12,6 \pm 9,4 anos de HAS. A pressão arterial média da população foi $150 / 84 \mathrm{mmHg}$. Os demais dados estão descritos na Tabela 1.

Tabela 1: Características clínicas e sociodemográficas da população ( $\mathrm{n}=65)$. Porto Alegre- RS, 2011.

\begin{tabular}{lc}
\hline Características & $\mathrm{n}(\%)$ \\
\hline Sexo feminino & $48(73,8)$ \\
Idade* $^{*}$ & $55,3 \pm 13,11$ \\
Tempo de hipertensão* & $12,6 \pm 9,4$ \\
Número de consultas* & $2,0 \pm 1,3$ \\
Escolaridade (anos completos de estudo)* $^{*}$ & $6,6 \pm 3,6$ \\
Situação conjugal (união estável) & $37(56,9)$ \\
Tabagismo & $5(7,7)$ \\
Diabetes & $18(27,7)$ \\
SAHOS & $21(32,3)$ \\
Depressão & $14(21,5)$ \\
IMC* & $30,6 \pm 7,0$
\end{tabular}

*Variável apresentada em média e desvio padrão. SAHOS: síndrome da apnéia hipopnéia obstrutiva do sono. IMC: Índice de massa corporal.

Os diagnósticos de enfermagem com maior prevalência e suas respectivas características definidoras foram Disposição para controle aumentado do regime terapêutico e Ansiedade (50,8\%), Estilo de vida sedentário (47,7\%) e Padrão de sono prejudicado $(46,4 \%)$, descritas na Tabela 2. 


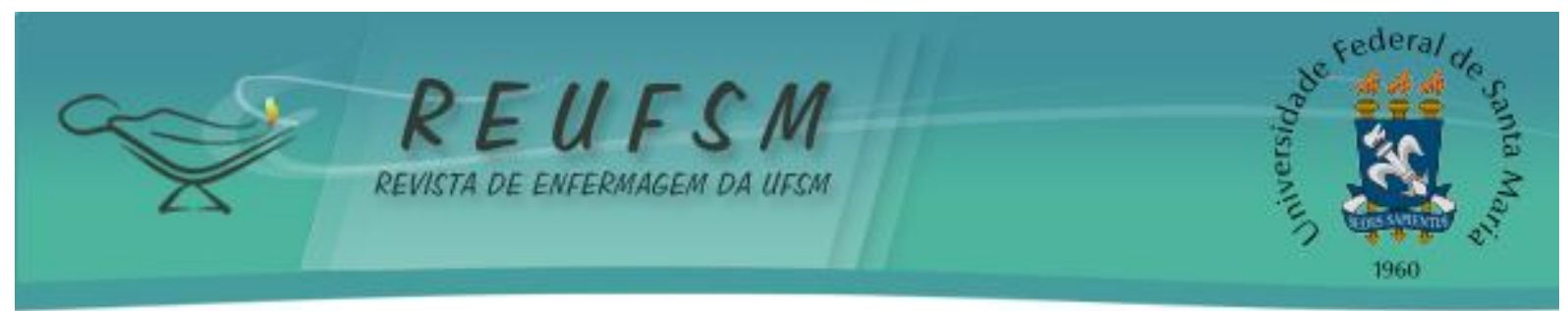

Tabela 2: Diagnósticos de enfermagem e suas características definidoras com alta prevalência, segundo domínios da NANDA-I em pacientes atendidos em ambulatório de hipertensão. Porto Alegre - RS, 2011.

Diagnóstico de Enfermagem e características definidoras
1) Disposição para controle aumentado do regime terapêtico

Descreve redução de fatores de risco

$33(50,8)$

Escolhas do dia-a-dia adequadas para o atendimento das metas

$9(13,8)$

Expressa desejo de controlar a doença

Expressa pouca dificuldade com o regime de tratamento prescrito

Não há aceleração inesperada dos sintomas da doença

$6(9,2)$

$3(4,6)$

2) Ansiedade

$33(50,8)$

Aflição

$18(27,7)$

Apreensão

Preocupação

$15(23,1)$

Tensão facial

$11(16,9)$

Insônia

3) Estilo de vida sedentário

Falta de condicionamento físico

Escolhe uma rotina diária sem exercícios físicos

$31(47,7)$

$25(38,5)$

Verbaliza preferência por atividades com pouco exercício físico

4) Padrão de sono prejudicado

Capacidade funcional diminuída

$30(46,4)$

$1(1,5)$

Insatisfação com o sono

$11(16,9)$

Mudanças no padrão normal do sono

$5(7,7)$

Queixa de não se sentir descansado

$11(16,9)$

Relato de dificuldade para dormir

$21(32,3)$

Os diagnósticos de enfermagem com média prevalência identificados nesta população foram Nutrição desequilibrada $(33,8 \%)$, Intolerância a atividade $(32,3 \%)$ e Volume de líquido excessivo (32,3\%). Os de baixa prevalência foram Falta de adesão $(15,4 \%)$, Risco de glicemia instável (13,8\%) e Disfunção sexual (13,8\%).

\section{DISCUSSÃO}

A HAS por ser uma patologia multifatorial e para que possa ser controlada, necessita da ação de vários profissionais para sua estabilidade e/ou resolutividade. Desta forma, requer do enfermeiro preparo na utilização da sistematização da assistência. A grande variedade de DE levantados na $1^{\text {a }}$ consulta em pacientes atendidos no ambulatório multiprofissional pode ser modificada com as intervenções de enfermagem, de acordo com o plano de ação individualizado para cada paciente. 


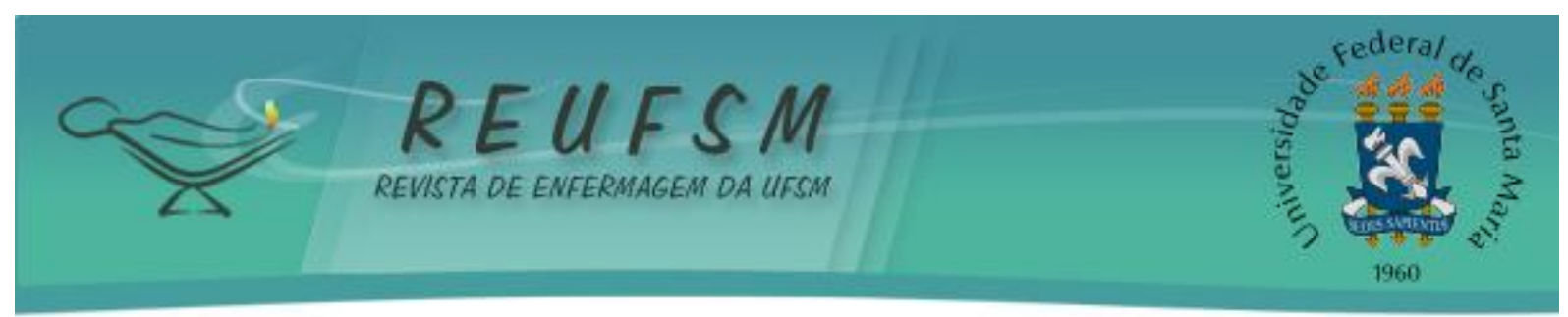

O diagnóstico de enfermagem Disposição para controle aumentado do regime terapêutico com o desejo expresso para controlá-lo, foi definido em metade dos pacientes deste estudo. Nesse sentido, a abordagem da enfermagem deve incluir recomendações acerca da necessidade de adesão ao tratamento, bem como reforçar as ações positivas dos pacientes, tendo em vista que a negligência pode acarretar sérios danos ao paciente.

A adesão dos pacientes hipertensos à terapêutica, no Brasil, é baixa e em 14 estudos populacionais realizados nos últimos quinze anos com 14.783 indivíduos (PA > $140 / 90 \mathrm{mmHg})$, revelaram baixos níveis de controle da PA $(19,6 \%) .{ }^{12} \mathrm{Em}$ um estudo transversal, de base populacional, cujo objetivo foi estimar a prevalência da HAS, assim como as características de seu controle e tratamento, em adultos de 18 a 90 anos, revelou que dos 1.003 indivíduos analisados, foi observada prevalência de HAS de $30,1 \%$, sendo que entre os hipertensos, $73,5 \%$ sabiam dessa condição, $61,9 \%$ faziam tratamento e apenas $24,2 \%$ tinham a PA controlada. ${ }^{20}$ Pode-se inferir que, apesar dos pacientes reconhecerem sua condição, tal fato não se reflete em ações efetivas de controle da doença.

Outro dado importante e determinante de negligência relacionada ao regime terapêutico refere-se à ocorrência da polifarmácia. Em um estudo que investigou os $\mathrm{DE}$ em idosos acamados no domicílio, evidenciou que $52 \%$ dos indivíduos utilizavam três e/ou mais medicamentos, com doses, apresentações e horários distintos do que foi prescrito. Alguns desses medicamentos têm efeitos colaterais que se somam, e outros que são adversos e agravam os déficits sensoriais, cognitivos e motores. Nesse estudo identificou-se que o elevado número de medicamentos e doses utilizados pelos idosos revela-se como um risco à saúde e à qualidade de vida, podendo gerar reações indesejadas relacionadas à complexidade do regime terapêutico. ${ }^{21}$ Tais evidências servem como alerta aos profissionais para a necessidade de mencionar aos pacientes que não basta somente ingerir os medicamentos, mas sim na dose, horário e frequência prescritos.

Neste estudo, o DE Ansiedade também foi atribuído para metade da amostra $(50,8 \%)$, evidenciando a interface de sintomas psicossociais com a doença cardiovascular. Esta característica psicológica é considerada como um traço de personalidade característico do indivíduo hipertenso. A investigação do funcionamento psicodinâmico desses sujeitos revela a existência de impulsos agressivos inibidos, concomitantes à presença de ansiedade. ${ }^{22}$ Em estudo de revisão, os trantornos do humor e de ansiedade têm sido associados a doenças cardíacas, no qual a HAS é uma das principais características encontradas. ${ }^{23}$

Outro DE presente em $46,4 \%$ dos hipertensos avaliados neste estudo foi o Padrão de sono prejudicado, sendo o relato de dificuldade para dormir associado à presença de despertares noturno causados pela necessidade de micções as principais evidências. $O$ uso de diurético e anti-hipertensivo ocasiona a necessidade do esvaziamento da bexiga, independente do horário. Da mesma forma, em um estudo com renais crônicos submetidos a transplante renal, que objetivou analisar as associações entre os $D E$, os fatores relacionados e as características definidoras descreve o DE Padrão de sono prejudicado relacionado à urgência urinária evidenciado por três ou mais despertares durante a noite como significativo nos pacientes investigados $(p=0,001) .{ }^{24}$

Outro DE que obteve destaque entre os pacientes deste estudo foi Estilo de vida sedentário sendo a falta de condicionamento físico a principal evidência. Entre as características da amostra, a média do IMC encontrado nos pacientes foi de 30,6 $\pm 7,0$, caracterizada como obesidade tipo $\mathrm{I}^{25}$ No mundo industrializado há um grande consumo de alimentos derivados da gordura saturada e no estilo de vida sedentário. A redução do gasto energético pela diminuição de atividade física habitual, associada à rotina da vida diária e ao aumento do tempo gasto em hábitos sedentários, tem levado as pessoas a se tornarem cada vez mais obesas. O exercício físico provoca gasto de energia pelo seu efeito direto 


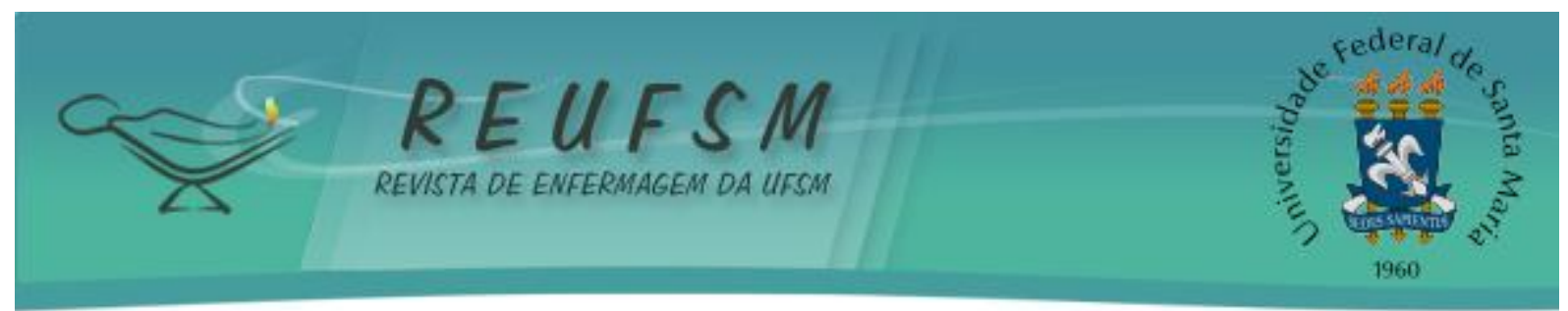

sobre o nível metabólico. No entanto, a dificuldade da prática do exercício físico entre os hipertensos obesos é uma realidade expressada na história clínica. A falta de ar e a dor nas articulações são sintomas que limitam, na maioria das vezes, a atividade física. Sendo assim, o exercício físico é uma prática que deve ser incorporada de uma forma lenta, gradual e sistemática. ${ }^{26}$

\section{CONCLUSÃO}

Os resultados encontrados neste estudo identificaram os diagnósticos de enfermagem prevalentes em pacientes hipertensos, possibilitando que a avaliação clínica do enfermeiro conduza à melhoria da qualidade dos cuidados de saúde. É necessário, no entanto avançar em estudos para a prescrição de cuidados e avaliação das intervenções educativas de enfermagem. Sendo assim a utilização dos DE poderá auxiliar a identificar a demanda terapêutica de autocuidado nos pacientes hipertensos com regime terapêutico complexo, fornecendo subsídios para a elaboração de um plano de ação baseado na resolutividade dos DE apontados.

\section{REFERÊNCIAS}

1. Gus I, Gus M, Zaslavsky C, Medina C, Harzheim E. Prevalência, reconhecimento e controle da hipertensão arterial sistêmica no estado do Rio Grande do Sul. Arq Bras Cardiol. [internet] 2004 nov [acesso em 2012 nov 29];83(5):424-8. Disponível em: http: / / www.scielo.br/scielo.php?script=sci_arttext\&pid=S0066-782X2004001700009.

2. Paiva DCP, Bersusa AAS, Escuder MML. Healthcare assessment for patients with diabetes and/or hypertension under the Family Health Program in Francisco Morato, São Paulo, Brazil. Cad Saúde Pública. [internet] 2006 Feb [cited 2012 Nov 29];22(2):377-85. Available from: http: / / www.scielo.br/scielo.php?pid=S0102-311X2006000200015\&script=sci_arttext .

3. Monteiro MF, Sobral Filho DC. Physical exercise and blood pressure control. Rev Bras Med Esporte. [internet] 2004 Nov/Dec [cited 2012 Nov 29];10(6):513-6. Available from: http: / /www.scielo.br/scielo.php?script=sci_arttext\&pid=S1517-

$86922004000600008 \&$ lang=pt.

4. Vidt DG. Pathogenesis and treatment of resistant hypertension. Minerva Med. 2003;94(4):201-4.

5. The Allhat Officers and Coordinators for the Allhat Collaborative Research Group. Major outcomes in high-risk hypertensive patients randomized to angiotensina converting enzyme inhibitor or calcium channel blocker vs. diuretic. The Antihypertensive and Lipid Lowering Treatment to Prevent Heart Attack Trial (ALLHAT). JAMA. 2002;288(23):2981-97.

6. Staessen JA, Fagard R, Thijs L, Celis H, Arabidze GG, Birkenhager WH, et al.

Randomized double-blind comparison of placebo and active treatment for older patients with isolated systolic hypertension. The Systolic Hypertension in Europe (Syst-Eur) Trial Investigators. Lancet. 1997;350(9080):757-64.

7. Lindhom LH, Ibsen H, Dahlof B, Edlman J, Ibsen H, Olsen M et al . Cardiovascular morbidity and mortality in patients with diabetes in the Losartan Intervention for Endpoint reduction nin hypertension study (LIFE): a randomized trial against atenolol. Lancet. 2002;359(9311):1004-10.

8. Goncalves SC, Silva E, Bertoluci C, Manfroi W, Fuchs FD. Hipertensão arterial nãocontrolada: causas e condutas. Rev Bras Hipertensão. 2005;8(2):56-9. 


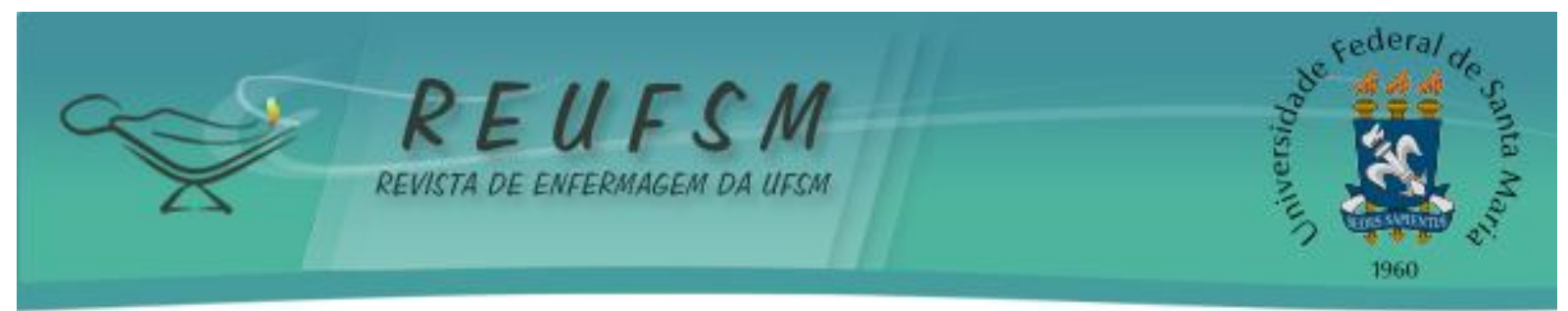

9. Osterberg L, Terrence B. Adherence to medication. N Engl J Med. 2005;353:487-97.

10. Silveira LMC, Ribeiro VMB. Compliance with treatment groups: a teaching and learning arena for healthcare professionals and patients. Interface comun saúde educ. [internet] 2005 Sept/Feb [cited 2012 Nov 29];9(16):91-104. Available from: http: / / www.scielo.br/scielo.php?script=sci_arttext\&pid=S1414-32832005000100008.

11. Braunwald E, Zipes Dp, Libby P. Tratado de Medicina Cardiovascular. $6^{\text {a }}$ ed. São Paulo: Roca; 2003.

12. Sociedade Brasileira de Hipertensão. VI Diretrizes Brasileiras de Hipertensão. Arq Bras Cardiol. [internet] 2010 [acesso em 2012 nov 29];95(1 Supl. 1):4-69. Disponível em: http: / / www.scielo.br/scielo.php?script=sci_arttext\&pid=S0066782X2010001700001\&lng=en\&nrm=iso\&tlng=pt .

13. Araújo TL, Maciel ICF, Maciel GGF, Silva ZMSA. Reflexo da hipertensão arterial no sistema familiar. Rev Soc Cardiol. 1998;2 (suppl A):1-6.

14. Andrade JS, Vieira MJ. Prática assistencial de enfermagem: problemas, perspectivas e necessidade de sistematização. Rev Bras Enferm. [internet] 2005 maio/jun [acesso em 2012 nov 29];58(3):261-5. Disponível em:

http: / / www.scielo.br/scielo.php?script=sci_arttext\&pid=S0034-71672005000300002.

15. Santos I, Figueiredo NMA, Duarte MJRS, Sobral VRS, Marinho AM. Enfermagem fundamental: realidade, questões e soluções. São Paulo (SP): Atheneu; 2002.

16. Silva LG, Jodas DA, Baggio SC, Vituri DW, Matsuda LM. Prescrição de enfermagem e qualidade do cuidado: um estudo documental. Rev Enferm UFSM. [internet] 2012 [acesso em 2012 nov 29];2(1):97-107. Disponível em: http://cascavel.ufsm.br/revistas/ojs2.2.2/index.php/reufsm/article/view/4546.

17. NANDA-I. Diagnósticos de Enfermagem da NANDA-I: definição e classificação 20092011/ NANDA-I International. Porto Alegre: Artmed; 2010.

18. Reppetto MA, Souza MF. Avaliação da realização e do registro da sistematização da assistência de enfermagem (SAE) em um hospital universitário. Rev Bras Enferm. [internet] 2005 [acesso em 2012 dez 05];58(3):325-9. Disponível em:

http: / / www.scielo.br/scielo.php?pid=S0034-71672005000300014\&script=sci_arttext.

19. Albuquerque JG, Lira ALBC, Lopes MVO. Fatores preditivos de diagnósticos de enfermagem em pacientes submetidos ao transplante renal. Rev Bras Enferm. [internet] 2010 [acesso em 2012 nov 29];63(1):98-103. Disponível em:

http: / / www.scielo.br/scielo.php?script=sci_abstract\&pid=S0034-

$71672010000100016 \&$ lng=en\&nrm=iso\&tlng=pt

20. Rosario TM, Scala LCNS, Franca GVA, Pereira MRG, Jardim PCBV. Prevalência, controle e tratamento da hipertensão arterial sistêmica em Nobres, MT. Arq Bras Card. [internet] 2009 dez [acesso em 2012 nov 29];93(6):672-8. Disponível em:

http: / / www.scielo.br/scielo.php?script=sci_abstract\&pid=S0066-

782X2009001200018\&lng=en\&nrm=iso\&tlng=pt.

21. Santos DN, Silva SNS, Silva DRS, Silva JC, Figueiredo MLF. Regime terapêutico inadequado em idosos acamados no domicílio. Rev Min Enferm. 2009;13 (2):177-82.

22. Wottrich SH, Ávila CM, Machado CC, Goldmeier S, Dillenburg D, Kuhl CP et al. Gênero e manifestação de stress em hipertensos. Estud psicol (Campinas). [internet] 2011 jan/mar 


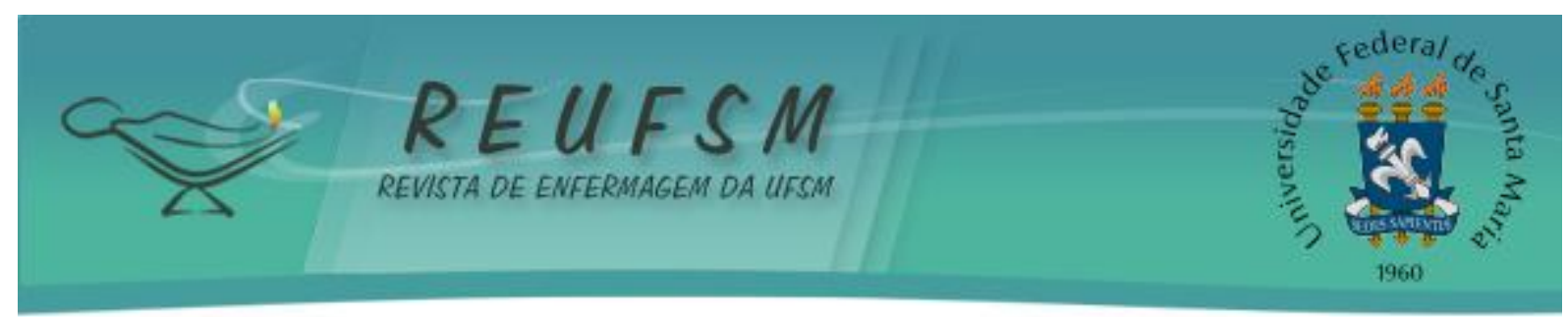

[acesso em 2012 nov 29];28(1):27-34. Disponível em:

http: / / www.scielo.br/scielo.php?script=sci_arttext\&pid=S0103-166X2011000100003.

23. Britta AL, Christenfeld NJS. Cardiovascular Disease and Psychiatric Comorbidity: ThePotential Role of Perseverative Cognition. Cardiovasc Psychiatry Neurol. 2009:1-8.

24. Lira ALBC, Lopes MVO. Pacientes transplantados renais: análise de associação dos diagnósticos de enfermagem. Rev Gaucha Enferm. [internet] 2010 mar [acesso em 2012 nov 29];31(1):108-14. Disponível em: http: //www.scielo.br/scielo.php?pid=S1983$14472010000100015 \&$ script $=$ sci_arttext.

25. World Health Organization. Obesity: preventing and managing the global epidemic. Report of a World Health Organization Consultation. Geneva: World Health Organization; 2000. p. 256. WHO Obesity Technical Report Series, n. 284.

26. Negrão CE, Trombetta IC, Tinucci T, Forjaz CLM. O papel do sedentarismo na obesidade. Rev Bras Hipertens. 2000;7(2):149-55.

Data de recebimento: 27/06/2012

Data de aceite: $27 / 12 / 2012$

Contato com autor responsável: Silvia Goldmeier

Endereço: Avenida Princesa Isabel, $370,3^{\circ}$ andar, Bairro Santana, Porto Alegre-RS.

CEP: $90620-000$

E-mail: pesquisa.sgold@gmail.com 